\title{
Monte Carlo studies of a novel X-ray tube anode design
}

\author{
L.M.N. Tavora ${ }^{\mathrm{a}}$, W.B. Gilboy ${ }^{\mathrm{b}, *}$, E.J. Morton ${ }^{\mathrm{b}}$ \\ ${ }^{a}$ Departamento de Fisica, Universidade de Coimbra, 3004-516 Coimbra, Portugal \\ ${ }^{\mathrm{b}}$ Department of Physics, University of Surrey, Guildford, Surrey GU2 $5 X H, U K$
}

\begin{abstract}
When energetic electrons are incident on high atomic number absorbers, a substantial fraction is back-scattered. This phenomenon is responsible for several undesirable effects in X-ray tubes, in particular a reduction in the X-ray output. The extent of this shortfall has been estimated by using Monte Carlo simulation to start electrons at increasing depth inside the anode, the results indicating that an output enhancement of nearly $50 \%$ could be achieved in principle if the electrons wasted in back-scatter events could be trapped inside a tungsten anode. To test this idea a further set of simulations were done for a novel anode geometry. Results showed that X-ray tube efficiencies might be substantially enhanced by this approach. (C) 2001 Elsevier Science Ltd. All rights reserved.
\end{abstract}

Keywords: X-ray tube; Anode design; Monte Carlo simulation

\section{Introduction}

The intense multiple scattering experienced by electrons due to the numerous electron-electron and electron-nucleus collisions as they slow down in matter gives rise to the phenomenon of electron back scattering from absorbers. For electrons with energies up to several hundred $\mathrm{keV}$ the back-scatter percentage in thick targets rises from about $2 \%$ in carbon to $50 \%$ in gold (Tabata et al., 1999). This property has been exploited for thickness gauging of sheet materials and surface coatings, but in other applications it is more problematic. In particular, in X-ray tubes, back-scattered electrons from high atomic number anode materials have several deleterious effects:

(i) Such electrons are absorbed by other parts of the tube structure leading to a large area secondary source of X-rays, which may diminish the quality of the spatial resolution in imaging applications.

(ii) Back-scattered electrons absorbed by the walls of the tube cause leakage currents across insulating components.

\footnotetext{
*Corresponding author. Tel.: +44-1483-876800; fax: +441483-876781.

E-mail address: w.gilboy@surrey.ac.uk (W.B. Gilboy).
}

(iii) Back-scattered electrons represent a loss of primary X-ray output from the anode.

This study addresses the last of these points by using a modified version of the EGS4 Monte Carlo code (Tavora and Morton, 1998) to simulate the transport of electrons and related radiations in a tungsten target. Initially a pencil beam of $120 \mathrm{keV}$ electrons was incident perpendicular to a $9 \mu \mathrm{m}$ thick tungsten target. This target thickness was found from previous work to maximise the total X-ray photon production (both bremsstrahlung and fluorescence) in transmission geometry.

In the present simulation study, electrons were started at different depths below the surface in the target, but the effective thickness of $9 \mu \mathrm{m}$ "seen" by the electrons in the forward direction was always the same. For example, in the case that the electron was due to "start" at a depth of $3 \mu \mathrm{m}$ in the target, the target thickness was defined to be $12 \mu \mathrm{m}$.

\section{Back-scattered electrons}

The amount of energy carried away by back-scattered electrons has long been studied, by both experimental 
and simulation approaches, for different target materials and beam energies. Two figures of merit are usually employed to describe electron back scattering. The energy of back-scattering coefficient $\left(\eta_{\mathrm{BE}}\right)$ is defined as the ratio of the energy carried away by back-scattered electrons to the total energy incident on the target. The fractional energy backscattering coefficient $\left(f_{\mathrm{BE}}\right)$ is a ratio of the mean energy of back-scattered electrons to that of incident electrons. In terms of judging how much of the energy is wasted, $\eta_{\mathrm{BE}}$ is more important, although higher values of $f_{\mathrm{BE}}$ indicate that back-scattered electrons have higher energies and so are more likely to produce additional $\mathrm{X}$-radiation if they can successfully be "trapped" inside the target.

Fig. 1 shows results for $\eta_{\mathrm{BE}}$. The data points are presented in the form $n 1_{-} n 2$, where $n 1$ corresponds to the W target thickness and $n 2$ the depth at which the electrons are started below the surface, with both values expressed in $\mu \mathrm{m}$. For example, the data point 12_3 corresponds to a set-up consisting of a $12 \mu \mathrm{m}$ target with the electrons "started" at a depth of $3 \mu \mathrm{m}$. As electrons are started deeper within the target, a smaller fraction of

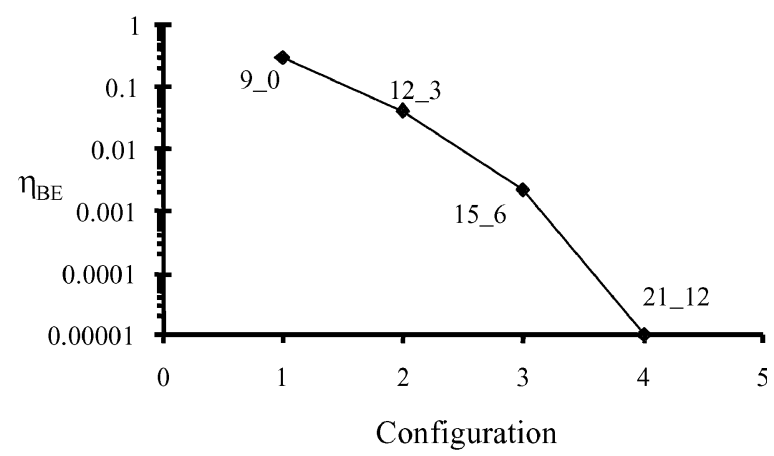

Fig. 1. Fraction of incident energy carried away by backscattered electrons for different target configurations.

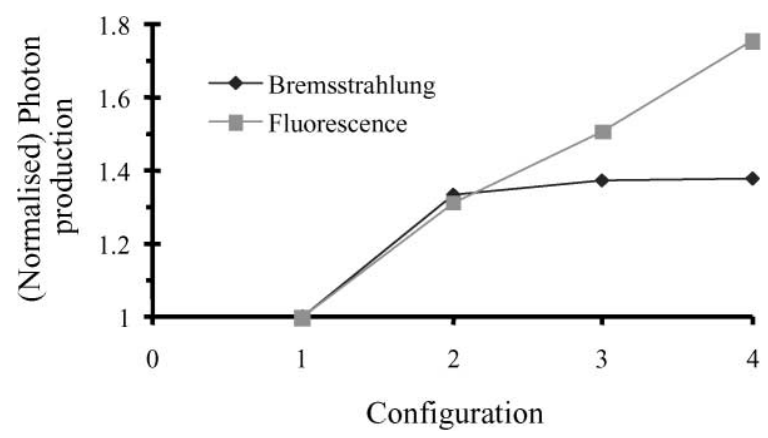

Fig. 2. Increase in photon production with starting depth in the target. energy is carried away by back-scattered electrons and results for $f_{\mathrm{BE}}$ show that these particles will also have a lower average energy.

\section{Photon production}

The production of photons within the target has also been evaluated for each configuration. Data for total photon production is shown in Fig. 2 for both bremsstrahlung and fluorescence radiation. The data points correspond to the target set-ups presented in Fig. 1, and the data was normalised to the first data point (i.e., the configuration 9_0 that corresponds to electrons being input at the surface). It is quite clear from this plot that there is a substantial enhancement in the production of X-radiation: almost $40 \%$ in terms of bremsstrahlung, and an even higher percentage increase in fluorescence as the starting depth increases.

The amount of radiation emitted from the two faces of the target has also been assessed. First, the total radiation flux emitted in the backward direction was logged and the results are shown in Fig. 3, with data sets corresponding to the total photon flux and fluorescence

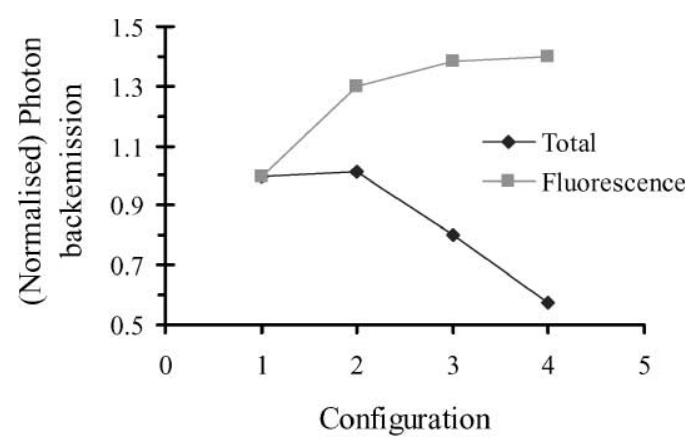

Fig. 3. Radiation back-emitted from the target.

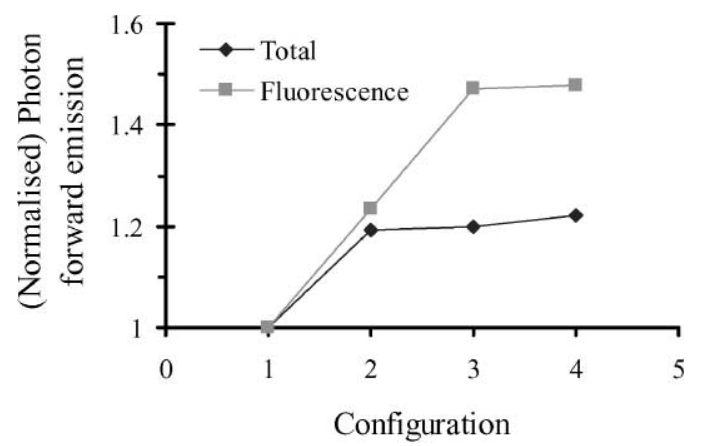

Fig. 4. Radiation emitted from the target in the forward direction. 
photons, respectively. Data is normalised to 9_0, i.e., electrons incident at the target surface. This plot shows that the number of fluorescence photons back-emitted from the target increases as electrons start deeper in the target, up to a depth of $12 \mu \mathrm{m}$, but the total flux decreases. This is a direct consequence of self-absorption in the target as electrons start further away from the "incident" surface, so they have an increased path on their way out.

Finally, the radiation emitted in the forward direction was also evaluated and the data is shown in Fig. 4. These data show that there is a clear and substantial enhancement of the radiation emitted in the forward direction, reaching a maximum fluorescence photon increase of about $47 \%$ at a start depth of $15 \mu \mathrm{m}$. Total photon production also increases by around $20 \%$.

These results are representative of further studies carried out for other target materials $(\mathrm{Ag}, \mathrm{Mo})$ and electron energies. For these cases too the gains obtained in terms of photon production increase with the energy of incident electrons.

\section{Conclusions}

These results suggest the possibility of enhancing the yield of fluorescence X-rays from an X-ray tube anode by modifying the anode geometry. Although impractical experimentally to start the electrons at depth in the target, it is nonetheless possible to pass the electrons down a narrow hole deep into the target. Simulations have been used to study this geometry, and data for electrons passed down a $10 \mu \mathrm{m}$ diameter hole into the target suggest that a clear enhancement of photon production can be obtained in both the forward and reverse directions.

\section{References}

Tabata, T., Andreo, P., Shinoda, K., 1999. Fractional energies of backscattered electrons and photon yields by electrons. Rad. Phys. Chem. 54 (1), 11-18.

Tavora, L.M.N., Morton, E.J., 1998. Photon production using a low energy electron expansion of the EGS4 code system. Nucl. Instrum. Methods B 143, 253-271. 